\title{
ABCC2(rs717620), and ABCC3 (rs4793665) affect high dose Methotrexate toxicity, and outcome in children with osteosarcoma
}

\author{
Amr Yahia ${ }^{1}$, Rania Labib ${ }^{2}$, Sameh A. Abdelshafi3², Asmaa Salama², Omneya Hassanain², \\ Hoda Rabea ${ }^{3}$, and Enas Elnadi ${ }^{2}$ \\ ${ }^{1}$ Pharm D; Faculty of Pharmacy Beni-Suef University, Teaching assistant Clinical \\ Pharmacy (Pharmacy Practice) Department; Faculty of Pharmacy, Horus University- \\ Egypt (HUE) \\ ${ }^{2}$ Children's Cancer Hospital Egypt 57357 \\ ${ }^{3}$ Beni Suef University Faculty of Pharmacy
}

December 22, 2020

\begin{abstract}
Objectives: Osteosarcoma (OS) is one of the aggressive bone tumors commonly diagnosed in adolescents and young adults. The study investigated the effect of 9 single nucleotide polymorphisms (SNPs) in 5 genes on methotrexate (MTX) plasma level and its impact on the patients' clinical outcomes. Methods: One hundred and thirty-three patients with OS (68 boys and 65 girls with age range 4.00-17.86 years) were enrolled and treated with four weeks of high-dose methotrexate (HDMTX) as neoadjuvant therapy in children's cancer hospital (CCHE-57357). Blood samples were collected and genotyped for the studied SNPs (MTHFR rs1801133, SLCO1B1 rs11045879, rs4149081, rs2306283, ABCC3 rs4793665, rs4148412, rs733392 ABCG2 rs2231142, and ABCC2 rs717620 using Taqman@ (RT-PCR) assay. Plasma concentrations of MTX were measured using the enzyme multiple immunoassay technique (EMITß). The clinical outcomes included toxicities that were evaluated based on the common terminology criteria for adverse events - version 5 (CTCAEv5.0.), besides the studying of survival analysis and tumor necrosis (TN \%). Results: Older patients experienced increased risk of delayed MTX elimination at 72 -hour, OR=1.19 (95\% CI=1.091.29, $\mathrm{p}=0.00059$ ). The studied SNPs weren't associated with MTX elimination. Patients with ABCC2 HW (Homozygous wild) genotype group showed a high statistically significant association with higher grade hyperbilirubinemia, OR $=2.05(95 \%$ $\mathrm{CI}=1.05-4.01, \mathrm{p}=0.037$ ). Moreover, the patients with ABCC3 (rs4793665) HV and HT (Homozygous variant and Heterozygous) genotypes had a significant association with severe nephrotoxicity, $\mathrm{OR}=0.34(95 \% \mathrm{CI}=1.6-0.72, \mathrm{p}=0.005)$. Conclusions: $\mathrm{ABCC} 2$ (rs717620), ABCC3 (rs4793665) genotyping with age and gender could help in predicting patients at risk of toxicities due to HDMTX.
\end{abstract}

ABCC2(rs717620), and ABCC3 (rs4793665) affect high dose Methotrexate toxicity, and outcome in children with osteosarcoma

Amr Y. Zakaria ${ }^{1^{*}}$; Rania M. Labib $2^{*}$; Sameh A. Abdelshafi ${ }^{3}$; Asmaa Salama ${ }^{4}$ Omneya Hassanain ${ }^{5}$ Hoda Rabea $^{6}$; Enas El Nadi ${ }^{7}$

1 Pharm D; Faculty of Pharmacy Beni-Suef University, Teaching assistant Clinical Pharmacy (Pharmacy Practice) Department; Faculty of Pharmacy, Horus University- Egypt (HUE).

2 Ph.D., Research Department, Children's Cancer Hospital-Egypt-57357, Cairo, Egypt.

3 Research Department, Children's Cancer Hospital-Egypt-57357, Cairo, Egypt.

4 Professor of Pathology, National Cancer Institute, Cairo University, Consultant of Pathology, Children's Cancer Hospital-Egypt-57357, Cairo, Egypt. 
5 Epidemiology and Biostatistics Unit, Research department, Children's Cancer Hospital-Egypt-57357, Cairo, Egypt.

6 Ph.D., Clinical Pharmacy Department, Faculty of Pharmacy Beni-Suef University, Egypt.

7 Assistant professor medical oncology department Faculty of Medicine Beni-Suef University, Consultant of pediatric oncology, Children's Cancer Hospital-Egypt-57357, Cairo, Egypt.

Full list of author information is available at the end of the article

* Authors contributed equally.

Correspondence: Rania M. Labib

Research Department, Children's Cancer Hospital

Address: 1 El-Emam Road, Sayeda Zeinab, Cairo, Egypt

Email:raniamlabib@gmail.com

Tel: +20225351500 extension: 7205; Fax: +20223619036 .

Location of study: Children's Cancer Hospital- 57357.

Acknowledgments: Not applicable.

Abstract

Objectives : Osteosarcoma (OS) is one of the aggressive bone tumors commonly diagnosed in adolescents and young adults. The study investigated the effect of 9 single nucleotide polymorphisms (SNPs) in 5 genes on methotrexate (MTX) plasma level and its impact on the patients' clinical outcomes.

Methods: One hundred and thirty-three patients with OS (68 boys and 65 girls with age range 4.00-17.86 years) were enrolled and treated with four weeks of high-dose methotrexate (HDMTX) as neo-adjuvant therapy in children's cancer hospital (CCHE-57357). Blood samples were collected and genotyped for the studied SNPs (MTHFR rs1801133, SLCO1B1 rs11045879, rs4149081, rs2306283, ABCC3 rs4793665, rs4148412, rs733392 ABCG2 rs2231142, and ABCC2 rs717620 using Taqman ${ }^{\circledR}$ (RT-PCR) assay. Plasma concentrations of MTX were measured using the enzyme multiple immunoassay technique (EMIT $\left.{ }^{\circledR}\right)$. The clinical outcomes included toxicities that were evaluated based on the common terminology criteria for adverse events - version 5 (CTCAEv5.0.), besides the studying of survival analysis and tumor necrosis (TN \%).

Results : Older patients experienced increased risk of delayed MTX elimination at 72-hour, OR=1.19 (95\% $\mathrm{CI}=1.09-1.29, p=0.00059)$. The studied SNPs weren't associated with MTX elimination. Patients with $A B$ CC2 $H W$ (Homozygous wild) genotype group showed a high statistically significant association with higher grade hyperbilirubinemia, $\mathrm{OR}=2.05(95 \% \mathrm{CI}=1.05-4.01, p=0.037)$. Moreover, the patients with $A B C C 3$ (rs4793665) $\mathrm{HV}$ and $H T$ (Homozygous variant and Heterozygous) genotypes had a significant association with severe nephrotoxicity, $\mathrm{OR}=0.34(95 \% \mathrm{CI}=1.6-0.72, p=0.005)$.

Conclusions : ABCC2 (rs717620), ABCC3 (rs4793665)genotyping with age and gender could help in predicting patients at risk of toxicities due to HDMTX.

Keywords : Osteosarcoma; Methotrexate; genotyping; ABCC2 (rs717620); ABCC3 (rs4793665) .

What's already known about this topic?

Osteosarcoma (OS) is one of the aggressive bone tumors commonly diagnosed in adolescents and young adults. The study investigated the effect of 9 single nucleotide polymorphisms (SNPs) in 5 genes on methotrexate (MTX) plasma level and its impact on the patients' clinical outcomes.

What does this article add? 
ABCC2 (rs717620), ABCC3 (rs4793665) genotyping with age and gender could help in predicting patients at risk of toxicities due to HDMTX.

\section{Introduction}

Osteosarcoma is one of the primary aggressive bone tumors often diagnosed with approximately $70 \%$ in children and adolescents (Hinds, Gattuso et al. 2009). The most frequent primary sites are the distal femur and proximal tibia. It is distinguished by mesenchymal spindle cells that deposit immature osteoid matrix (Serra and Hattinger 2017). The Rizzoli Institute studies outlined a crucial role of MTX, cisplatin, and doxorubicin as the most active chemotherapeutic agents for OS. The study by (Lui, Treluyer et al. 2018) highlighted the evidence that 3-year disease-free survival rates of $60-70 \%$ following the administration of preoperative multi-agent chemotherapy followed by surgical resection and maintenance chemotherapy. European and American osteosarcoma study group (EURAMOS) protocol is an international randomized study on patients with OS, which HDMTX $\left(12 \mathrm{~g} / \mathrm{m}^{2}\right)$ infused over 4-hour per week as neo-adjuvant chemotherapy for four weeks (Goricar, Kovac et al. 2014, Hattinger, Tavanti et al. 2017). MTX is an antineoplastic agent and prescribed in various cancer treatment protocols such as acute leukemia, breast cancer, and OS (Giletti and Esperon 2018). MTX competitively inhibits certain folate-dependent enzymes and the biosynthesis of DNA nucleotides such as purines, pyrimidines, and encoded proteins resulting in apoptosis. Extracellular MTX is transported to the cell by various pathways, primarily through active transport mediated by reduced folate carriers (RFCs). Passive diffusion adds to the transfer of MTX by cellular inflow. Whereas the majority of MTX is eliminated by renal excretion, with less than $20 \%$ biliary secretion (Howard, McCormick et al. 2016, Giletti and Esperon 2018). MTX is cellular transmitted by the solute carrier organic anion transporters family member 1B1 (SLCO1B1). On the other hand, ABC genes that encode p-glycoprotein are responsible for the efflux of MTX out of the cell, such as human multidrug resistance-related protein 2 (MRP2), encoded by ABC subfamily C member2 (ABCC2). Methylene-tetrahydrofolate Reductase (MTHFR) 677C $>$ T rs1801133 located at the coding region of the gene. Several studies thus far have linkedMTHFR SNP with toxicity and clinical outcome (Gregers 2012, Lambrecht, Sleurs et al. 2017, Giletti and Esperon 2018). The beta elimination in the MTX terminal decay curve has a useful predictor for MTX elimination kinetics. HDMTX toxicities may include hematological toxicity, hepatotoxicity, and nephrotoxicity as it has narrow therapeutic index drug (NTI) (López López 2013, Goricar, Kovac et al. 2014, Serra and Hattinger 2017, Giletti and Esperon 2018).

Inter-individual variations in age, gender, and genetic makeup can cause decreased effectiveness of drug therapy, or side effects, or toxicity, or treatment failure. According to the American Society of HealthSystem Pharmacists (ASHP), " Pharmacogenetics (PGs) investigates the effect of genomic variations on drug responses. PGs testing can improve drug response that may include sub-optimal clinical outcomes, decreased cost of treatment, better medication adherence, more appropriate selection of therapeutic agents, decreased length of treatment, and enhanced patient safety "(Swen, Nijenhuis et al. 2011, Manolio, Chisholm et al. 2013, Johnson 2016). Single nucleotide polymorphisms (SNPs) is the leading cause of genetic variations accounted for about 90\% of the human genome (Feuk, Carson et al. 2006). It may influence the role or expression of the gene, the inter-individual differences in pharmacokinetics (PKs), and pharmacodynamics (PDs) parameters (Przekop Jr, Tulgan et al. 2006). The current study aimed to determine the allelic frequencies of 9 SNPs in 5 genes (MTHFR, SLCO1B1, ABCC3, ABCG2, and ABCC2)involved in the MTX pathway in the children with OS. Our study investigated the role of PGs variations on MTX elimination, toxicities, survival, and TN\%. Hopefully, this will help us in the future to build models that accurately predict drug response and toxicity for individual patients and use this knowledge to personalize treatment plans. 


\section{Materials and methods}

\section{Design and participants}

A retrospective cohort study included 133 patients diagnosed with localized non-metastatic OS of the extremity treated based on the EURAMOS protocol at CCHE-57357 between 2013 and 2018 in Cairo, Egypt. The study was approved by the Institutional Review Board (IRB). All patients and their parents or legal guardians have signed written informed consent. Patients were included if they fit the following eligibility criteria.

\section{Patient inclusion criteria}

Newly diagnosed non-metastatic localized resectable disease, primary OS, histological evidence of high-grade OS of an extremity (femur, tibia, humerus, fibula, radius and ulna), age [?] 18 years at the date of the diagnostic biopsy, started chemotherapy within 30 days of diagnostic biopsy, and adequate bone marrow function; neutrophils count $>1.5 \times 10^{9} / \mathrm{L}$ (or WBC $>3 \times 10^{9} / \mathrm{L}$ if neutrophils were not available) and platelet count $>100 \times 10^{9} / \mathrm{L}$., renal function (glomerular filtration rate [?]70 ml $/ \mathrm{min} / 1.73 \mathrm{~m}^{2}$ ), and liver function (total bilirubin [?]1.5 $\times$ upper limit of normal (ULN)).

\section{Patient exclusion criteria}

Any previous treatment for OS and any medical history of chemotherapy, juxtacortical (periosteal, parosteal) OS, unresectable primary disease, or metastatic, or both, and if patients administered any drug that can affect MTX level that would be recorded.

\section{Clinical protocol}

All patients treated with HDMTX (12000 mg $/ \mathrm{m}^{2} \mathrm{I} . \mathrm{V}$ over 4-hour infusion), administered at weeks $(4,5,9$, and 10) as per protocol. The leucovorin rescue (LR) of $15 \mathrm{mg} / \mathrm{m}^{2} / \mathrm{q}$ 6-hour started 24-hour after the initiation of the infusion of HDMTX (El Mesallamy, Rashed et al. 2014, Goricar, Kovac et al. 2014). The patients 'data collected from Cerner (electronic medical records) using (RED-Cap?), a web-based data collection tool (Harris, Taylor et al. 2009).

\section{Genotyping}

Blood samples (5ml) were collected for genotyping. Genomic DNA was extracted using the Gene-JET kits (Taqman $^{(\mathrm{r})}$ - Thermo- fisher scientific Baltics UAB V, Lithuania). DNA concentrations were detected using Nano-Quant Infinite M200 PRO (Tecan Group Ltd., Switzerland). Genotyping carried out using QuantStudio 12 K Flex Real-Time PCR System Life Technologies Ltd (Paisley PA4 9RF, UK). The predesigned and drug-metabolizing enzymes (DME) studied genes SNPs processed according to (Thermo- fisher scientific Baltics protocol). The volumes of plate wells didn't exceed $10-\mu \mathrm{L}$ for the cycling system. Life Technologies software was adapted for allele discrimination analysis (de ROTTE, Bulatovic et al. 2012).

\section{Plasma level analysis of MTX}

The analyses of MTX levels were carried out after 24-hour infusion initiation, and every 24-hour, until the MTX level was [?]0.2 $\mu \mathrm{mol} / \mathrm{L}$ (Howard, McCormick et al. 2016, Lui, Treluyer et al. 2018). This was measured by (EMIT ${ }^{\circledR}$ MTX 2011), supplied with Siemens Healthcare Diagnostics Inc., SYVA ${ }^{\circledR}$, USA (El Mesallamy, Rashed et al. 2014). 


\section{Outcome analysis}

TN\% followed preoperative chemotherapy was histopathological determined. All patients were assessed for HDMTX toxicities and were graded and recorded according to (CTCAE v5.0) (Goricar, Kovac et al. 2014, Lui, Treluyer et al. 2018). 5-year overall survival (O.S) and event-free survival (EFS) were analyzed.

\section{Statistical analysis}

The patients were divided into two groups according to genotyping results: group 1 was $(H W)$, and group 2 included patients with at least one variant allele $(H V$ plus $H T)$. Patients' levels of MTX at 72-hour were categorized to "good elimination" (72-hour $<0.2 \mu \mathrm{Mol} / \mathrm{L}$ ) or "delayed elimination" (72-hour [?] $0.2 \mu \mathrm{Mol} / \mathrm{L}$ ) (Martinez, Muhrez et al. 2018). To account for the correlated nature of the cumulative data, the generalized linear mixed model (GzLMM) fit by maximum likelihood (Laplace Approximation) for binomial data was used to test the association between SNPs and patterns of MTX plasma levels at 72-hour. The measured toxicities were divided into two types moderate (grade 1-2) and severe (grade 3-4). It followed (CTCAE v5.0) guidelines for hematological toxicity, hepatotoxicity, and nephrotoxicity (Goricar, Kovac et al. 2014, Lui, Treluyer et al. 2018, Martinez, Muhrez et al. 2018). TN, if [?] 90\% considered to be poor responders as having more than $10 \%$ viable tumor at the time of surgery, and $>90 \%$ good responders (Hattinger, Tavanti et al. 2017, Serra and Hattinger 2017). Cumulative logit link function for ordinal outcomes used to examine the relationship between SNPs and toxicities to determine the significance of the selected variables and to estimate effect sizes (Pinheiro and Bates 2006). 5-years O.S and EFS were estimated using Kaplan - Meier. The log-rank test analyzed the study differences in the estimates of endpoint events over time concerning the SNPs investigated. Hazard ratios and $95 \%$ confidence intervals (CIs) were calculated by Cox proportional hazards regression model for univariate and multivariate analyses to identify independent predictors of O.S and EFS (both SNPs and demographic variables).

\subsubsection{Statistical methods}

Quantitative variables were summarized using the range, mean, and standard deviation. The frequency and percentage were used for qualitative variables. The analysis was done using $\mathrm{R}$ for statistical computing $\mathrm{R}$ version 3.6.1. Significance was considered at $p[?] 0.05$.

\section{Results}

\subsection{Patients' demographics}

One hundred and thirty-three patients were eligible and included in this study. There were 68 boys $(51.1 \%)$ and 65 girls $(48.9 \%)$. The mean age at diagnosis was $12.51 \pm 3.33$ years, and the median was 13.06 years (range, 4.00-17.86 years). The mean body surface area was $1.4 \pm 0.31 \mathrm{~m}^{2}$. Primary tumor sites were femur, tibia, fibula, humerus, radius, and ulna representing $(57.8 \%, 33.1 \%, 4.5 \%, 3 \%, 0.75 \%$, and $0.75 \%$ respectively). TN\% was poor responders and good responders representing $(67.5 \%$ and $32.5 \%$ respectively). The survival status was alive, dead and loss of follow up representing $(79.9 \%, 18.8 \%$, and $1.5 \%$ respectively) The main characteristics of the patients were included in (Table 1).

\subsection{Genotyping}

The results of genotyping and allelic frequencies are listed in (Table 2). The minor allele frequency (MAF) for MTHFR (rs1801133)C > T, SLCO1B1 (rs4149081) A > G,ABCG2 (rs2231142) G> T, ABCC2 (rs717620) $C>T$, and $A B C C 3$ (rs4148412) $\mathrm{T}>\mathrm{C}$ were $(0.29,0.49,0.27,0.11$, and 0.42 , respectively). While forSLCO1B1 (rs11045879) C> T, SLCO1B1 (rs2306283) A> G, ABCC3 (rs4793665) C> T, and $A B$ CC3 (rs733392) G> A, the variant allele was more predominant with an allelic frequency of $(0.63,0.53$, 0.60 , and 0.85 , respectively).

\subsection{Genotypes-MTX level analysis}


Association between the two patients' groups of each gene SNP and delayed MTX pattern at 72-hour MTX levels is illustrated in (Table 3). Only the age was significantly associated with delayed elimination at 72 hour. A one-year increase in patients' age was associated with a $19 \%$ increase in the risk of MTX delayed excretion, $\mathrm{OR}=1.19(95 \% \mathrm{CI}=1.09-1.29, p=0.00059)$.

\subsection{Genotypes-toxicity analysis}

The age has significantly associated with neutropenia, as increasing the age by one-year decreases the expected severe neutropenic risk by $9 \%, \mathrm{OR}=0.91(95 \% \mathrm{CI}=0.84-0.99, p=0.02)$. Male patients were less likely to develop anemia compared to females, $\mathrm{OR}=0.47(95 \% \mathrm{CI}=0.27-0.84, p=0.009)$. Older patients were less likely to develop higher grades of enzymatic hepatotoxicity for (ALT), OR=0.88(95\% CI=0.8-0.95, $p=0.002)$, and $(\mathrm{AST}), \mathrm{OR}=0.82(95 \% \mathrm{CI}=0.76-0.89, p=0.001)$. Male patients were at higher risk for the development of clinically severe hyperbilirubinemia following administration of HDMTX, OR=1.53(95\% CI=1.04-2.24, $p$ $=0.03$ ). When ABCC2 (rs717620) was statistically adjusted for age, the results showed that patients with the same-sex having $H W$ genotype group ( $80.5 \%$ of the studied population) were significantly associated with a $105 \%$ increase in the risk of hyperbilirubinemia, $\mathrm{OR}=2.05(95 \% \mathrm{CI}=1.05-4.01, p=0.037)$. The findings showed that every year old was associated with a $10 \%$ increase in the risk of nephrotoxicity, $\mathrm{OR}=1.1(95 \%$ $\mathrm{CI}=1.03-1.2, p=0.009)$. Patients with at least one variant alleles (group 2) of ABCC3 (rs4793665) (86\% of the studied population) had higher risk to develop severe nephrotoxicity, OR=0.31(95\% $\mathrm{CI}=0.14-0.67$, $p=0.003$ ). When $A B C C 3$ (rs4793665)statistically adjusted for age for the same-sex, it was still significantly associated with the risk to progress severe nephrotoxicity in patients of group 2, OR=0.34(95\% CI=1.6-0.72, $p=0.005)$. Association between the two patients' groups of each gene SNP, age, gender, and different severe toxicities after four HDMTX cycles is illustrated in (Table 4).

\subsection{Genotypes-outcome analysis}

None of the SNPs have significantly associated with TN\% or O.S and EFS, as shown in (Figure 1).

\section{Discussion}

This study examined the emerging role of the pharmacogenetic markers in children with OS to minimize inter-individual variations in chemotherapy such as HDMTX. The objectives of many studies are to design prognostic tools for protection against any potential toxicity resulting in treatment failure, prolongation of hospitalization, and decrease of survival. It was achieved by studying the relation between PGs involving gene SNPs, drug PKs, and PDs. The problem here, many studies had divergent, conflicting findings and a lack of reproducibility, a small number of homogeneous ethnicities, variations in ethnic groups, differences in treatment protocols, varied assessment of toxicity criteria, and clinical outcomes (López López 2013, LopezLopez, Gutierrez-Camino et al. 2014). The present study offered essential findings on a retrospective cohort of patients larger than published in prior similar studies. It provided an in-depth analysis of a homogeneous Egyptian ethnicity with the same pre-therapy conditions. They had treated with the same protocol, reliable, well-recorded data as well as distinguished objective toxicity criteria, survival, and TN\% analysis.

The most important clinically relevant finding was the reliability of MTX terminal elimination pattern at 72-hour. The relationship based on the studies of (Treviño, Shimasaki et al. 2009, López López 2013) on HDMTX in pediatric acute lymphoblastic leukemia (ALL). Therefore, our study relied on an objective and quantifiable marker that was cutoff 72-hour MTX level. Regarding the allelic frequency determination, the significant finding was that the variant allele was more predominant with an allelic frequency of $S L C O 1 B 1$ (rs11045879), SLCO1B1 (rs2306283), ABCC3 (rs4793665), and ABCC3 (rs733392) in Egyptian children with OS. Recent developments in the field of PGs have led to a renewed interest in the polymorphic effect of transporter and metabolism genes on PKs and PDs of MTX.

In the current study, a one-year increase in age-associated with a 19\% increased risk of MTX delayed elimination pattern at 72-hour. So, older age children were considered poor eliminators of HDMTX in the 
studied sample. The interpretation of our results may be the low volume of distribution of MTX. The young children have high body water content leading to more MTX elimination than elder children. These results match those observed in earlier studies of (Csordas, Hegyi et al. 2013, Luetke, Meyers et al. 2014, Wippel, Gundle et al. 2019, Young, Cheng et al. 2020). The study of (Csordas, Hegyi et al. 2013) reported the significant association between high levels in children age older than 14 years because the metabolism resembles those of adult patients. The younger children were less likely to have delayed elimination due to their physiology. The findings of the present study further support the idea of different genotypes did not have a significant association with level as consistent with (Csordas, Hegyi et al. 2013, El Mesallamy, Rashed et al. 2014). Csordas, Hegyi et al. (2013) explained the insignificant association that six-fold higher MTX dose in OS than ALL. It led to an increase in the activity of ABC -transporters genes of liver and kidney, so it enhanced the excretion of MTX toxic metabolites like 7-hydroxy-MTX and MTX excretion.

Prior studies demonstrated that the factors related to the appearance of certain types of toxicities after HDMTX administration. These factors may be due to higher MTX levels after multiple HDMTX cycles or other factors. The previous studies offered contradictory findings about several factors participate in this association such as; (Hoekstra, Van Ede et al. 2003, Li, Sui et al. 2019, Mandal, Samaddar et al. 2020, Young, Cheng et al. 2020). MTX level wouldn't predict appearance specific type of toxicity except nephrotoxicity after multiple HDMTX cycles, described by (Tsurusawa, Gosho et al. 2015). Mandal, Samaddar et al. (2020) revealed, MTX levels didn't associate with toxicity. He explained that gender, body mass index (BMI), and the pre-cycles ANC made that association. Besides, basic serum creatinine was the predictor of toxicity. The study of (Vaishnavi, Bansal et al. 2018) in low and middle-income countries at a single center in (Chandigarh, India) recommended that the safe to give full HDMTX to ALL patients with extended hydration, additional doses of LR, monitoring of serum creatinine, and $\mathrm{pH}$ without therapeutic drug monitoring (TDM) of MTX.

Regarding hematological toxicity risk, the present study showed severe neutropenia, which was a statistically significant association with young children as consistent with (Ferrari, Palmerini et al. 2009, Young, Cheng et al. 2020). Male gender was less likely to associate with severe anemia as compared to females due to normal physiology as consistent with (Ferrari, Palmerini et al. 2009). In the Ferrari, Palmerini et al. (2009) study provided a guide for neutropenic risk prediction, significantly increased in young children (4-14 years) and female gender. The beneficial application of that study is to give a young female patient a higher dose of granulocyte colony-stimulating factor (G-CSF) post HDMTX cycles to decrease neutropenia risk. The variability might relate to the difference in gender sensitivity to chemotherapy. The study of (Young, Cheng et al. 2020) confirmed our findings that severe neutropenia had associated with younger children with low body surface area. The current results offered some essential insights into the absence of a significant other hematological toxicity with studied gene SNPs in agreement with (Yang, Wang et al. 2013, Suthandiram, Gan et al. 2014, Membrive, Ramírez et al. 2020). Suthandiram, Gan et al. (2014) had noted that ABCG2 polymorphism didn't associate with toxicity. So, the recommendations of our study are to translate our findings to the patients' bedsides as preventive measures. The present study results proved that the older age and female patients were at high risk of hematological toxicity.

This study provides an exciting opportunity to advance our knowledge of polymorphism of ABC transporter genes. Recently investigators have examined the effects of ABCC2 (rs717620) SNP on toxicity. ABCC2

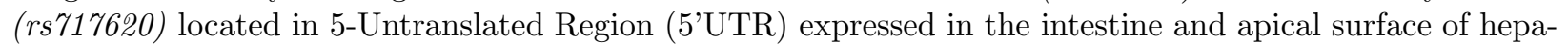
tocytes (Hegyi, Arany et al. 2017). Patients with the same-sex in group 1 (80.5\% of the tested population) withABCC2 (rs717620) SNP were associated with double risk to develop severe hyperbilirubinemia agreed with (Goricar, Kovac et al. 2014, Hattinger, Tavanti et al. 2017). Interestingly, the current study proved that male patients had a $53 \%$ increase in the risk to have significant severe grade hyperbilirubinemia. So, the cumulative risk (153\%) for severe hyperbilirubinemia would arise for male patient with ABCC2 (rs717620) SNP $H W$ genotype. The interpretation of our findings may due to the greater accumulation of MTX toxic concentrations in hepatocytes causing severe grade hyperbilirubinemia patients in group 1 with $A B C C 2$ (rs717620) SNP $H W$ genotype. They have impaired hepatic clearance and decreased volume of distribution of HDMTX, as consistent with the findings of (Simon, Marsot et al. 2013). Younger children have a higher risk for significant severe elevation in liver enzymes, consistent with (Holmboe, Andersen et al. 2012). Most 
patients were exposed to temporary and reversible increases in serum (ALT) and (AST) values, which may not result in chronic liver disease following administration of HDMTX (Howard, McCormick et al. 2016). The screening of ABCC2 (rs717620)polymorphism especially for male patients and young children could help in preventing severe hepatotoxicity risk.

As far as $A B C C 3$ located in the gall bladder, and kidney. The expression of $A B C C 3$ mRNA is associated with drug resistance. Its biological function is organic anion excretion like MTX and bile (Liu, Yi et al. 2014). Children in group 2 having ABCC3 (rs4793665) (86\% of the tested population) were associated with an increase in the risk of severe nephrotoxicity even after adjusting for age. The findings were inconsistent with (de ROTTE, Bulatovic et al. 2012, Hegyi, Arany et al. 2017). The results of Hegyi, Arany et al. (2017) study demonstrated that the presence of at least one variant allele T in patients with ABCC3 (rs4793665) causes higher MTX concentrations than wild-type allele C, and the polymorphism affects the outcome due to altering its binding to the nuclear factor. Consequently, our findings explain that patients in group 2 with at least one variant allele T may cause impaired excretion of MTX, 7-hydroxy-MTX, and inactive 4amino-4-deoxy-N-methylpteroic acid (DAMPA). The solubilities of these metabolites in urine may reach ten folds lower than MTX (Schmiegelow 2009). The sequel may cause renal tubules obstruction, and extended contact of MTX with the renal tubular epithelial tissue lining exposes it to direct toxic harm and acute kidney injury(AKI) (Perazella and Moeckel 2010). LR cannot prevent nephrotoxicity(Li, Sui et al. 2019), so Patients in group 2 who are at greater risk of irreversible acute kidney failure are expected to increase their serum creatinine by more than 50\% within 24-hour (Howard, McCormick et al. 2016). The strong point in our study has a large sample size that proved the toxicity associated with SNPs, as consistent with recommendations of.(de ROTTE, Bulatovic et al. 2012) In the present study, older age was associated with a 10\% increase in risk every year for higher grade renal toxicity after HDMTX, agreed with (Csordas, Hegyi et al. 2013, May, Carson et al. 2014, Fabresse, Devictor et al. 2018). May, Carson et al. (2014) clarified patients at risk of significant nephrotoxicity; older age patients, and decreased baseline creatinine clearance. Our study proved that the nephrotoxicity risk could be prevented by ABCC3 (rs4793665) SNP screening, and elder age children determination.

The current study offered some essential insights into the absence of the significant association of TN\% with different gene SNPs, which were consistent with previous publications like Serra (Serra and Hattinger 2017). Eventually, the study has gone some way towards enhancing our understanding of the clinical outcome of survival analysis by The Kaplan - Meier estimation for the studied SNPs. The observed difference between O.S and EFS with SNPs genotypes in this study hadn't reached a significant level. Further work is required to establish this association.

\section{Conclusion}

To sum up, our study provided a reference for clinicians to enhance the understanding of the preventive measures of toxicity that may be associated with HDMTX in Egyptian children with OS. Factors and candidate biomarkers could affect toxicity and HDMTX elimination by the concurrent analysis of PGs and other factors like age and gender.

\subsection{Importance in practice}

For the future best clinical practice, the present study recommended that: (1) Physicians should take care of dosing MTX in older children to avoid delayed elimination and high risk of severe nephrotoxicity. (2) Screening patients for ABCC2 (rs717620) SNP: 80.5\% of Egyptian children in the study with $H W$ genotype have clinically severe hyperbilirubinemia and increased by a $53 \%$ in male patients. (3) A reversible increase in liver enzymes could associate with younger children decreased by using drugs supporting hepatic cells. (4) Screening patients for ABCC3 (rs4793665) SNP: 86.0\% of Egyptian children in the study with at least one variant allele and the older ages have an associated risk of irreversible severe nephrotoxicity. (5) MTX dose adjustment should be according to the patient's genetic profile, sex, and age. It is a retrospective cohort 
study of patients with a larger sample size than published in prior similar studies.

5.2 Study limitations

There are some limitations to be noted. The possibility of having other unknown factors affects PKs and PDs of HDMTX. Our findings are limited to our hospital center, as the analysis had relied on a singlecenter. Unfortunately, the available previous mapped data are limited, which requires further investigations in Egyptian children with OS.

\section{Declarations}

6.1 Author contributions Amr Zakaria: Sample collection/consenting, Lab work, manuscript writing, (budget 6 SNPs) -

Rania Labib: Idea, Design of work, provided training/supervision for lab work, arranging sample collection/ acquisition, all administrative approvals (for IRB. Study team, SMAC), manuscript writing/ revision, (budget 3 SNPs) -

Hoda Rabea: Revision and administrative work for faculty_Sameh Abdelshafi: Lab work_ Enas Elnadi: Clinical data revision_ Omneya Hassanain: Statistical analysis/Results interpretation.

6.2 Funding: own fund.

6.3 Data availability: The data that support the findings of this study are available from the corresponding author upon request.

\subsection{Compliance with Ethical Standards}

6.5 Conflict of interest: The authors declare that they have no conflicting interests.

6.6 Ethical approval: This study was approved by the Institutional Review Board (25-June-2018) at CCHE-57357.

6.6.1. Consent for publication: All authors have consented to submission of this article for publication.

6.6.2. Consent to participate: All participants in the original trials or their parent/legal guardian signed a document of informed consent and assent was obtained as appropriate.

6.7 location of the study: Children's Cancer Hospital-57357(CCHE-57357), Cairo, Egypt.

\section{References}

Csordas, K., M. Hegyi, O. T. Eipel, J. Muller, D. J. Erdelyi and G. T. Kovacs (2013). "Comparison of pharmacokinetics and toxicity after high-dose methotrexate treatments in children with acute lymphoblastic leukemia." Anti-Cancer Drugs 24 (2): 189-197.

de ROTTE, M. C., M. Bulatovic, M. W. Heijstek, G. Jansen, S. G. Heil, R. H. van SCHAIK, N. M. Wulffraat and R. de JONGE (2012). "ABCB1 and ABCC3 gene polymorphisms are associated with first-year response to methotrexate in juvenile idiopathic arthritis." The Journal of rheumatology 39 (10): 2032-2040.

El Mesallamy, H. O., W. M. Rashed, N. M. Hamdy and N. Hamdy (2014). "High-dose methotrexate in Egyptian pediatric acute lymphoblastic leukemia: the impact of ABCG2 C421A genetic polymorphism on plasma levels, what is next?" Journal of cancer research and clinical oncology 140 (8): 1359-1365.

Fabresse, N., B. Devictor, C. Pissier, H. Chambost, B. Lacarelle, G. Michel and C. Solas (2018). "Plasma 7-Hydroxymethotrexate Levels Versus Methotrexate to Predict Delayed Elimination in Children Receiving High-Dose Methotrexate." Therapeutic drug monitoring 40 (1): 76-83. 
Ferrari, S., E. Palmerini, E. Staals, M. Abate, A. Longhi, M. Cesari, A. Balladelli, L. Pratelli and G. Bacci (2009). "Sex-and age-related chemotherapy toxicity in patients with non-metastatic osteosarcoma." Journal of chemotherapy 21 (2): 205-210.

Feuk, L., A. R. Carson and S. W. Scherer (2006). "Structural variation in the human genome." Nature Reviews Genetics 7 (2): 85-97.

Giletti, A. and P. Esperon (2018). "Genetic markers in methotrexate treatments." The pharmacogenomics journal : 1 .

Goricar, K., V. Kovac, J. Jazbec, B. Zakotnik, J. Lamovec and V. Dolžan (2014). "Influence of the folate pathway and transporter polymorphisms on methotrexate treatment outcome in osteosarcoma." Pharmacogenetics and genomics 24 (10): 514-521.

Gregers, J. (2012). "Pharmacogenetic studies in childhood acute lymphoblastic leukaemia with primary focus on methotrexate."

Harris, P. A., R. Taylor, R. Thielke, J. Payne, N. Gonzalez and J. G. Conde (2009). "Research electronic data capture (REDCap) - a metadata-driven methodology and workflow process for providing translational research informatics support." Journal of biomedical informatics 42 (2): 377-381.

Hattinger, C. M., E. Tavanti, M. Fanelli, S. Vella, P. Picci and M. Serra (2017). "Pharmacogenomics of genes involved in antifolate drug response and toxicity in osteosarcoma." Expert opinion on drug metabolism 83 toxicology 13 (3): 245-257.

Hegyi, M., A. Arany, A. F. Semsei, K. Csordas, O. Eipel, A. Gezsi, N. Kutszegi, M. Csoka, J. Muller and D. J. Erdelyi (2017). "Pharmacogenetic analysis of high-dose methotrexate treatment in children with osteosarcoma." Oncotarget8 (6): 9388.

Hinds, P. S., J. S. Gattuso, C. A. Billups, N. K. West, J. Wu, C. Rivera, J. Quintana, M. Villarroel and N. C. Daw (2009). "Aggressive treatment of non-metastatic osteosarcoma improves health-related quality of life in children and adolescents." European Journal of Cancer 45 (11): 2007-2014.

Hoekstra, M., A. Van Ede, C. Haagsma, M. Van De Laar, T. Huizinga, M. Kruijsen and R. Laan (2003). "Factors associated with toxicity, final dose, and efficacy of methotrexate in patients with rheumatoid arthritis." Annals of the Rheumatic Diseases 62 (5): 423-426.

Holmboe, L., A. M. Andersen, L. Mørkrid, L. Slørdal and K. S. Hall (2012). "High dose methotrexate chemotherapy: pharmacokinetics, folate and toxicity in osteosarcoma patients." British journal of clinical pharmacology 73 (1): 106-114.

Howard, S. C., J. McCormick, C.-H. Pui, R. K. Buddington and R. D. Harvey (2016). "Preventing and managing toxicities of high-dose methotrexate." The oncologist21 (12): 1471-1482.

Howard, S. C., J. McCormick, C.-H. Pui, R. K. Buddington and R. D. Harvey (2016). "Preventing and managing toxicities of high-dose methotrexate." The oncologist : theoncologist. 2015-0164.

Johnson, J. A. (2016). "Pharmacists should jump onto the clinical pharmacogenetics train." American Journal of Health-System Pharmacy 73 (23): 2013-2016.

Lambrecht, L., C. Sleurs, V. Labarque, C. Dhooge, A. Laenen, F. Sinnaeve, M. Renard and A. Uyttebroeck (2017). "The role of the MTHFR C677T polymorphism in methotrexate-induced toxicity in pediatric osteosarcoma patients." Pharmacogenomics 18 (8): 787-795.

Li, X., Z. Sui, F. Jing, W. Xu, X. Li, Q. Guo, S. Sun and X. Bi (2019). "Identifying risk factors for high-dose methotrexate-induced toxicities in children with acute lymphoblastic leukemia." Cancer Management and Research11 : 6265 . 
Liu, S., Z. Yi, M. Ling, J. Shi, Y. Qiu and S. Yang (2014). "Predictive potential of ABCB1, ABCC3, and GSTP1 gene polymorphisms on osteosarcoma survival after chemotherapy." Tumor Biology 35 (10): 98979904.

Lopez-Lopez, E., A. Gutierrez-Camino, N. Bilbao-Aldaiturriaga, M. Pombar-Gomez, I. Martin-Guerrero and A. Garcia-Orad (2014). "Pharmacogenetics of childhood acute lymphoblastic leukemia." Pharmacogenomics 15 (10): 1383-1398.

López López, E. (2013). "New genetic markers for treatment personalization in pediatric Acute Lymphoblastic Leukemia."

Luetke, A., P. A. Meyers, I. Lewis and H. Juergens (2014). "Osteosarcoma treatment-where do we stand? A state of the art review." Cancer treatment reviews 40 (4): 523-532.

Lui, G., J. M. Treluyer, B. Fresneau, S. Piperno-Neumann, N. Gaspar, N. Corradini, J. C. Gentet, P. Marec Berard, V. Laurence and P. Schneider (2018). "A pharmacokinetic and pharmacogenetic analysis of osteosarcoma patients treated with high-dose methotrexate: Data from the OS2006/Sarcoma-09 trial." The Journal of Clinical Pharmacology 58 (12): 1541-1549.

Mandal, P., S. Samaddar, J. Chandra, N. Parakh and M. Goel (2020). "Adverse effects with intravenous methotrexate in children with acute lymphoblastic leukemia/lymphoma: a retrospective study." Indian Journal of Hematology and Blood Transfusion: 1-7.

Manolio, T. A., R. L. Chisholm, B. Ozenberger, D. M. Roden, M. S. Williams, R. Wilson, D. Bick, E. P. Bottinger, M. H. Brilliant and C. Eng (2013). "Implementing genomic medicine in the clinic: the future is here." Genetics in Medicine15 (4): 258-267.

Martinez, D., K. Muhrez, J. B. Woillard, A. Berthelot, E. Gyan, S. Choquet, C. R. Andres, P. Marquet and C. Barin-Le Guellec (2018). "Endogenous Metabolites-Mediated Communication Between OAT1/OAT3 and OATP1B1 May Explain the Association Between SLCO1B1 SNPs and Methotrexate Toxicity." Clinical Pharmacology 83 Therapeutics 104 (4): 687-698.

May, J., K. R. Carson, S. Butler, W. Liu, N. L. Bartlett and N. D. Wagner-Johnston (2014). "High incidence of methotrexate associated renal toxicity in patients with lymphoma: a retrospective analysis." Leukemia E lymphoma 55 (6): 1345-1349.

Membrive, C., C. P. Ramirez, N. M. Pete, M. M. Montoro, S. A. Santiago, A. J. Morales and M. R. Tortosa (2020). "5PSQ-065 Prediction of toxicity of methotrexate by means of genetic tests in patients diagnosed with moderate-severe psoriasis."

Perazella, M. A. and G. W. Moeckel (2010). Nephrotoxicity from chemotherapeutic agents: clinical manifestations, pathobiology, and prevention/therapy . Seminars in nephrology, Elsevier.

Pinheiro, J. and D. Bates (2006). "Mixed-effects models in S and S-PLUS."

Przekop Jr, P. R., H. Tulgan, A. A. Przekop and M. Glantz (2006). "Adverse drug reaction to methotrexate: pharmacogenetic origin." Journal of the American Osteopathic Association 106 (12): 706.

Schmiegelow, K. (2009). "Advances in individual prediction of methotrexate toxicity: a review." British journal of haematology 146 (5): 489-503.

Serra, M. and C. Hattinger (2017). "The pharmacogenomics of osteosarcoma." The pharmacogenomics journal 17 (1): 11.

Simon, N., A. Marsot, E. Villard, S. Choquet, H. Khe, N. Zahr, P. Lechat, V. Leblond and J. Hulot (2013). "Impact of ABCC2 polymorphisms on high-dose methotrexate pharmacokinetics in patients with lymphoid malignancy." The pharmacogenomics journal 13 (6): 507. 
Suthandiram, S., G.-G. Gan, S. M. Zain, P.-C. Bee, L.-H. Lian, K.-M. Chang, T.-C. Ong and Z. Mohamed (2014). "Effect of polymorphisms within methotrexate pathway genes on methotrexate toxicity and plasma levels in adults with hematological malignancies." Pharmacogenomics 15 (11): 1479-1494.

Swen, J., M. Nijenhuis, A. de Boer, L. Grandia, A.-H. Maitland-van der Zee, H. Mulder, G. Rongen, R. Van Schaik, T. Schalekamp and D. Touw (2011). "Pharmacogenetics: from bench to byte - an update of guidelines." Clinical Pharmacology $\mathcal{E}$ Therapeutics 89 (5): 662-673.

Trevino, L. R., N. Shimasaki, W. Yang, J. C. Panetta, C. Cheng, D. Pei, D. Chan, A. Sparreboom, K. M. Giacomini and C.-H. Pui (2009). "Germline genetic variation in an organic anion transporter polypeptide associated with methotrexate pharmacokinetics and clinical effects." Journal of Clinical Oncology 27 (35): 5972 .

Tsurusawa, M., M. Gosho, T. Mori, T. Mitsui, S. Sunami, R. Kobayashi, R. Fukano, F. Tanaka, N. Fujita and H. Inada (2015). "Statistical analysis of relation between plasma methotrexate concentration and toxicity in high-dose methotrexate therapy of childhood nonHodgkin lymphoma." Pediatric blood $\&$ cancer 62 (2): 279-284.

Vaishnavi, K., D. Bansal, A. Trehan, R. Jain and S. V. Attri (2018). "Improving the safety of high-dose methotrexate for children with hematologic cancers in settings without access to MTX levels using extended hydration and additional leucovorin." Pediatric blood \& cancer 65 (12): e27241.

Wippel, B., K. R. Gundle, T. Dang, J. Paxton, J. Bubalo, L. Stork, R. Fu, C. W. Ryan and L. E. Davis (2019). "Safety and efficacy of high-dose methotrexate for osteosarcoma in adolescents compared with young adults." Cancer medicine 8 (1): 111-116.

Yang, J., Z.-G. Wang, H.-Q. Cai, Y.-C. Li and Y.-L. Xu (2013). "Effect of variation of ABCB1 and ABCC3 genotypes on the survival of bone tumor cases after chemotherapy." Asian Pacific Journal of Cancer Prevention 14 (8): 4595-4598.

Young, E. P., W. S. Cheng, M. B. Bernhardt, L. L. Wang, N. Rainusso and J. H. Foster (2020). "Risk factors associated with delayed methotrexate clearance and increased toxicity in pediatric patients with osteosarcoma." Pediatric Blood \& Cancer 67 (4): e28123.

\section{Hosted file}

figures IJCP15-12-2020.pdf available at https://authorea.com/users/384404/articles/500025abcc2-rs717620-and-abcc3-rs4793665-affect-high-dose-methotrexate-toxicity-and-outcomein-children-with-osteosarcoma

\section{Hosted file}

Tables(IJCP15-12-2020).pdf available at https://authorea.com/users/384404/articles/500025abcc2-rs717620-and-abcc3-rs4793665-affect-high-dose-methotrexate-toxicity-and-outcomein-children-with-osteosarcoma 